Voix et Images

voixetimages

\title{
Variation, fragmentation, répétition
}

Pascal Riendeau

Volume 40, numéro 1 (118), automne 2014

URI : https://id.erudit.org/iderudit/1028031ar

DOI : https://doi.org/10.7202/1028031ar

Aller au sommaire du numéro

Éditeur(s)

Université du Québec à Montréal

ISSN

0318-9201 (imprimé)

1705-933X (numérique)

Découvrir la revue

Citer ce compte rendu

Riendeau, P. (2014). Compte rendu de [Variation, fragmentation, répétition].

Voix et Images, 40(1), 178-183. https://doi.org/10.7202/1028031ar

Ce document est protégé par la loi sur le droit d'auteur. L'utilisation des services d'Érudit (y compris la reproduction) est assujettie à sa politique d'utilisation que vous pouvez consulter en ligne.

https://apropos.erudit.org/fr/usagers/politique-dutilisation/ 


\author{
ROM A N \\ Variation, fragmentation, répétition \\ $+++$
}

PASCAL RIENDEAU

Université de Toronto

Variations endogènes ${ }^{1}$, la plus récente publication de Karoline Georges, explore de multiples univers où il est question du corps, de l'intériorité, en insistant surtout sur la sexualité humaine. Il convient d'emblée de souligner un choix éditorial: la photographie de la couverture, Means of Reproduction no. 1192 de l'artiste Svjetlana Tepavcevic, affiche une très jolie feuille de la koelreuteria elegans, qui suggère bien la thématique sans l'imposer. Pour Karoline Georges, il s'agit moins de raconter les joies ou les passions de l'érotisme que de montrer des univers où dominent les obsessions, les perversions, les bizarreries. En quatorze textes composés pour la plupart comme des nouvelles de facture classique, Karoline Georges explore des conceptions insolites de la sexualité et de l'attachement amoureux. L'auteure maîtrise bien l'art de ciseler les textes courts, en plus de posséder une très grande habileté à créer des chutes ou des renversements inattendus. Quand l'ironie ne domine pas, l'humour se fait parfois léger ou, au contraire, noir, voire sordide. Dans la majorité des textes, cela s'inscrit dans ce que l'on pourrait appeler une narration non sentimentale, en ce sens qu'aucune situation, si dramatique soit-elle, n'est traitée du point de vue des sentiments ou même des émotions morales. L'aspect détaché de la narration de nombreuses nouvelles la rend beaucoup plus précise - elle peut paraître assez sèche - et convient parfaitement à ces univers où les excès et les débordements priment. En revanche, d'autres nouvelles, narrées à la première personne, misent sur la sentimentalité de manière exagérée, sous-entendant ainsi une distance ironique entre l'auteure et le narrateur. Malgré les comportements ou les discours étranges des personnages au sein de ces fictions brèves, les mondes qu'ils habitent doivent être qualifiés, par convention, de réalistes. On en sort toutefois dans la dernière nouvelle, «L'ordre du jour», la seule dans laquelle les raisons qui conduisent à la situation fantaisiste relèvent plutôt de variations exogènes.

La première nouvelle, «La victime», donne le ton en racontant une histoire sombre où il est question de prostitution juvénile. Mais la victime n'est peut-être pas celle que l'on pense, ou alors celle que le récit semble désigner d'emblée comme victime ne s'avère assurément pas la seule. Bien que la compassion ou la honte

1 Karoline Georges, Variations endogènes, Québec, Alto, 2014, 153 p. 
restent totalement absentes, une légère tendance moralisatrice n'échappe pas à la conclusion. «L'incitation», la nouvelle suivante, illustre bien la capacité de l'auteure à reprendre une variation sur un thème éculé dans la nouvelle - le suicide comme conclusion inéluctable au récit -, afin de lui donner une pertinence supplémentaire. Réécrire la scène de suicide poignante n'est pas une sinécure, mais Karoline Georges y parvient avec beaucoup d'efficacité et de précision. Encore une fois, l'absence totale de sentimentalité et de larmoiement rend la finale plus juste. L'incursion du côté des formes les plus surprenantes de la sexualité se trouve sans doute dans la nouvelle «L'amour». Le narrateur, un homme immensément riche qui écrit une lettre testamentaire à Laura, relate la relation fusionnelle et parfaite qu'il a avec elle. Cette longue déclaration d'amour laisse difficilement entrevoir que Laura n'était pas réellement une femme, mais une poupée de taille humaine qui ressemble de manière presque stupéfiante à une vraie personne. Contrairement à celui de la majorité des autres textes, le style impose une certaine grandiloquence. Le détachement ne se trouve pas dans le texte lui-même, mais entre la nouvelliste et le personnage principal. On entre ici dans le registre dont parle Jean-Marie Schaeffer à propos des romans $d u$ romanesque, c'est-à-dire des œuvres qui préconisent une «distanciation (souvent ironique), donc une dissonance entre l'auteur et le personnage ${ }^{2} »$. Il semble difficile de ne pas considérer les élans de lyrisme du protagoniste comme dérisoires:

Avant toi Laura, j'incarnais le fantasme des amazones, qui paradaient devant moi dans leur suite de poses, de sourires, d'intonations ponctuées de petits rires enfantins pour titiller ma testostérone. [...] Avant toi Laura, j'étais devenu un pied de nez pour l'ensemble de l'humanité qui n'avait plus d'autre réalité pour moi que son potentiel boursier. [...] Avant toi Laura, j'éjaculais comme on se ronge les ongles. Avec compulsion, à répétition, sans trop m’en rendre compte. (23-25)

Cette recherche d'un style élevé se précise quand le narrateur, dans ses dernières volontés, demande "qu'un poète réécrive» la lettre que nous venons de lire "pour lui insuffler toute la noblesse nécessaire» (31). Peut-on vraiment être amoureux d'un objet manufacturé qui ressemble tellement à une personne et qui peut procurer à celui qui la côtoie un plaisir sexuel inouii?

Les nouvelles qui ne portent pas de manière spécifique sur la sexualité exploitent néanmoins les bizarreries ou les obsessions des relations intimes et familiales. À l'instar de la nouvelle "L'amour», «L'autoportrait» consiste en un long discours de la protagoniste sans que l'on puisse avoir accès aux paroles ou aux pensées de son interlocuteur. La variation endogène prend un sens plus littéral dans ce texte. Après les suicides successifs de sa mère et de son père, la narratrice, qui vivait dans un musée-mausolée conçu par celui-ci en hommage à celle-là, choisit d'aller encore plus loin dans son désir de fusion avec ses parents: «J'ai patienté huit ans. Ā me remémorer nos longues périodes d'attente, qui ravivaient notre passion familiale. [...] Puis mon père a respecté sa promesse. Ils sont enfin revenus. Enfin presque.

2 Jean-Marie Schaeffer, «La catégorie du romanesque», Gilles Declercq et Michel Murat (dir.), Le romanesque, Paris, Presses Sorbonne Nouvelle, 2004, p. 297. 
Nous partageons maintenant mon corps.» (113) Jusqu'où peut-on aller afin de ressembler à celle que l'on admire?

Les variations Burroughs ${ }^{3}$ de Sylvie Nicolas propose une forme beaucoup plus expérimentale. Roman fragmenté, roman-variations au sens où le suggérait Kundera à propos du Livre du rire et de l'oubli ${ }^{4}$, Les variations Burroughs est composé d'un dispositif narratif assez complexe. Elles-mêmes divisées en quelques récits, les huit variations principales (ou chapitres) procurent au texte une forme très morcelée. Les différents récits, qui portent autant sur le passé que sur le présent de la narratrice, s'entrecroisent si finement que l'on ne peut pas déterminer lequel est le principal. La narratrice glisse aussi dans son roman des extraits d'un journal tenu de manière aléatoire qu'elle appelle "Ma vie sans Charles» (son ancien amoureux), mais sans trop insister sur la rupture. Dans ce va-et-vient constant entre les récits et les époques, $c^{\prime}$ est davantage la relation au frère qui domine l'espace psychologique du texte. Ce frère, qui est interpellé dès l'incipit, elle le décrit comme "le gardien de la mémoire» (16). Il est aussi question d'un deuxième frère - le frère mort ou le «bébé-frère — puis d'un troisième qui a failli mourir dans un incendie: «on ne savait toujours pas ce qu'il fallait inventer pour sauver un frère» (18).

Le livre de Sylvie Nicolas s'ouvre sur l'image d'une boîte de livres «trouvée aux ordures» (9) offerte à la narratrice par le frère à qui elle s'adresse dans le roman. La métaphore du contenant rempli d'un trésor ou de souvenirs familiaux se prolonge par la valise qui l'accompagnera toute sa vie dans ses multiples déplacements. Ces livres qu'elle a reçus assez jeune lui ouvrent la porte vers un monde inconnu, presque insoupçonnable. La narratrice affirme avoir lu à douze ans L'amant de Lady Chatterley, Un tramway nommé désir ou Des souris et des hommes. La rencontre avec l'univers de William Burroughs, qui reste associé à celui de Charles, se produira beaucoup plus tard. Truffé de citations et de références diverses, Les variations Burroughs laisse une belle place à la parole des autres. Lectrice de Nancy Huston, de Jacques Prévert, de Jacques Poulin, de Milan Kundera et d'une quantité d'autres auteurs, romanciers et essayistes, la narratrice s'inspire de leurs idées, les intègre à ses pensées et s'en sert pour tenter de comprendre les événements et les personnes qui l'entourent.

La présence de Burroughs comme sujet de conversation entre les personnages ou comme objet de réflexion de la part de la narratrice paraît à la fois constante et diffuse. Si la fragmentation peut rappeler les romans de l'auteur emblématique de la beat generation, on reste très loin de l'imitation. Tout en s'intéressant au romancier, la narratrice critique l'homme et avoue avoir des réticences à son égard d'un point de vue moral: comment peut-on admirer l'auteur qui a tué Joan Vollmer, qui était alors sa femme? À un ami de Charles qui lui demande comment elle souhaite

3 Sylvie Nicolas, Les variations Burroughs, Montréal, Éditions Druide, coll. «Écarts», 2014, 175 p.

4 «Tout ce livre est un roman en forme de variations. Les différentes parties se suivent comme les différentes étapes d'un voyage qui conduit à l'intérieur d'un thème, à l'intérieur d'une pensée, à l'intérieur d'une seule et unique situation dont la compréhension se perd pour moi dans l'immensité», Milan Kundera, Le livre du rire et de l'oubli, traduit du tchèque par François Kérel, Paris, Gallimard, coll. «Folio», 1998 [1978], p. 254. 
que l'on traite l'œuvre de Burroughs, elle répond de manière indirecte, préférant se lancer dans un mouvement introspectif: "Je ne savais pas comment faire pour continuer à tenir entre mes mains ce qui s'était écrit avec le sang des autres.» (76) Toutes les discussions autour de Burroughs l'incitent à replonger dans une biographie de l'auteur qu'elle décrit comme non autorisée, sans doute celle de Ted Morgan ${ }^{5}$. Dans My Education, texte fragmentaire autobiographique publié deux ans avant sa mort, Burroughs affirme: "Ted Morgan's biography starts with a basic misconception: Literary Outlaw. To be an outlaw, you must first have a base in law to reject and get out of. I never had such a base ${ }^{6}$.» Lors d'un autre échange avec des écrivains et artistes, alors qu'elle se trouve en résidence d'écriture à Banff, la narratrice fait part de sa conception du rôle qu'aurait joué Burroughs dans la littérature américaine. À sa grande surprise, son opinion se trouve corroborée par Tony Allard, un artiste qui a eu l'occasion de rencontrer le romancier peu de temps avant sa mort, et qui a produit une œuvre vidéographique étrange: Corpse and Mirror (1995).

Présenté à l'origine à l'Université Laval en 2013 comme mémoire de maîtrise en création littéraire sous le titre "Les variations Burroughs ou l'équation de Dieu», le texte de Sylvie Nicolas a conservé des éléments réflexifs propres aux travaux universitaires, notamment autour de la question de l'archive fictionnelle (169), tout en réussissant parfaitement la transition vers sa forme publiée. À la fin du roman, la narratrice devient autoréflexive comme lorsqu'elle décrit son projet: "Vous cédez à la tentation de créer un texte, une série de tableaux qui s'achèveraient sur un coup de feu, un verre qui roule sur le plancher [...]; une suite de variations que vous vous plaisez à qualifier de "burroughs".» (158) La variation comme forme se révèle d'autant plus pertinente pour la narratrice qu'elle crée un lien essentiel avec sa propre vie: «je suis issue d'une suite de variations» (167). Très nuancé, le roman de Sylvie Nicolas n'est pas sans posséder une certaine pudeur également, comme si se dévoiler, pour la narratrice, ne signifiait pas tout dire, mais bien ne révéler que quelques éléments essentiels.

Auteur de fictions, blogueur, fondateur de l'Académie de la vie littéraire, Mathieu Arsenault fait paraître à l'hiver 2014 La vie littéraire ${ }^{7}$. Au même moment, son éditeur précédent republie Album de finissants ${ }^{8}$, dans lequel on trouvait déjà une structure fragmentée similaire. Récit bref, frénétique, La vie littéraire ne favorise toutefois qu'une seule voix narrative plutôt que les multiples voix d'Album de finissants.

5 Ted Morgan, Literary Outlaw. Life and Times of William S. Burroughs, New York, W. W. Norton, 1988, 768 p.

6 William S. Burroughs, My Education. A Book of Dreams, New York, Viking Penguin, 1995, p. 7. «La biographie de Ted Morgan est basée sur une prémisse erronée: Hors-la-loi littéraire. Pour être un hors-la-loi, on doit d'abord posséder un fondement à l'intérieur de la loi que l'on peut rejeter et quitter. Je n'ai jamais eu un tel fondement. " Je traduis.

7 Mathieu Arsenault, La vie littéraire, Montréal, Le Quartanier, coll. «Série QR», 2014, 103 p.

8 Mathieu Arsenault, Album de finissants, Montréal, Triptyque, 2014 [2004], 143 p. Cette édition en format de poche a paru au moment où une adaptation théâtrale du texte a été présentée au Théâtre DenisePelletier dans une mise en scène d'Anne Sophie Rouleau. 
Précédées d'un court préambule et d'une conclusion brève pour le moins déroutante, les trois grandes sections, intitulées «Lire», «Écrire» et "Imprimer», sont composées de plus de soixante-dix fragments aux titres parfois inusités: "Pisser dans son bain», "Josée Yvon princesse du désert» ou encore glissant du côté d'une vulgarité puérile: "Plotte plotte Nietzsche», «Basketball diarrhée». La vie littéraire est liée de près à Vickie Gendreau et au dernier texte qu'elle a écrit juste avant sa mort, Drama Queens. C'est d'ailleurs Mathieu Arsenault qui lui a servi de lecteur privilégié et d'éditeur pour ce livre publié à titre posthume. Jeune femme dans la vingtaine, la narratrice de La vie littéraire, ce court récit fragmenté racontant des moments de vie hachurés, n'est pas sans rappeler l'auteure de Testament ${ }^{9}$. Pas plus que Sylvie Nicolas à l'égard de Burroughs, Arsenault n'a cherché à imiter la voix ou le style de Vickie Gendreau, mais bien à créer un certain rapprochement esthétique avec elle.

La vie littéraire possède une signature stylistique forte: ponctuation minimale, usage des majuscules fortement restreint, langage qui relève de l'oralité. Cela implique aussi une importante création de néologismes, une adaptation de mots usuels ou encore une abondance d'expressions anglaises, comme dans le fragment «Le lancement de la nouvelle saison»:

\begin{abstract}
le vin à volonté fuck ouais dans des verres en plastique fuck ouais j'en ai renversé sur des livres yolo je l'ai dit à personne cool story bro j'étais tellement saoule cool story bro j'étais tellement gênée yolo cool story bro j'en ai acheté un autre cool story mais je l'ai oublié sur une étagère [...] il n’y a pas de temps pour se relire seulement pour aller dans les lancements acheter les livres taper un petit poème self-loathing de marde [...]. (52)
\end{abstract}

Arsenault a réussi à créer une langue singulière qui combine les univers des réseaux sociaux, des jeux vidéo, du nouveau franglais montréalais, mais entrecoupée de passages d'un style plus soutenu. Le ton de la narratrice est souvent frondeur, railleur - elle affirme qu'elle aurait "voulu écrire ce glorieux livre de poche» (17) qui se trouve dans le corpus d'étude de l'épreuve uniforme de français, mais le récit donne l'impression qu'une quête se profile derrière le sarcasme. Il reste difficile de savoir quoi exactement, mais la narratrice révèle un désir de créer, de s'affirmer. Malgré son ironie constante, on sent chez elle une admiration réelle pour des textes de Kathy Acker, de France Théoret ou de Julie Doucet.

Dans les longues phrases répétitives et sans ponctuation, on trouve de très nombreux commentaires de la narratrice sur son écriture. À l'intérieur de ses multiples coups de gueule ou de ses élucubrations, son parti pris pour la littérature - et plus particulièrement le livre qu'elle souhaite écrire - demeure l'élément clé. Il est éloquent dans ce passage, où l'on peut percevoir en filigrane le programme d'Arsenault:

non ce ne sera pas un roman non ce ne sera pas une grande fresque historique non plus un article journalistique non plus un texte d'opinion non plus une entrée de

9 Vicky Gendreau, Testament, Montréal, Le Quartanier, coll. «Série QR», 2012, 159 p. 
blog non plus une lettre non plus rien ce sera n'importe quoi ça va être drôle ça va être triste ça va être grandiose et ça va être laid et ordurier mais oh que non ce ne sera plus jamais communiquer [...]. (43)

Privilégier la fragmentation ne crée pas que des zones de liberté pour les auteurs. On sent parfois dans chacun des textes l'application de procédés, voire l'imposition de contraintes, notamment chez Mathieu Arsenault. En revanche, c'est bien grâce à une structure plus répétitive qu'il réussit à donner une parole forte à sa protagoniste. Dans sa version plus classique, la variation de Karoline Georges nous plonge au cœur de ce qui constitue l'étrangeté de la sexualité humaine ou de ce qui nous transforme de l'intérieur. Pour Sylvie Nicolas, le sens de la variation reste plus formel. Chez l'une comme chez l'autre, la variation se présente comme une exploration d'univers ou de situations multiples, mais qui n'exclut pas la répétition. 